\title{
Age-dependency of the serum oxidative level in the senescence-accelerated mouse prone 8
}

\author{
Sakiko TANIGUCHI ${ }^{1}$, Masakazu HANAFUSA ${ }^{2}$, Hirokazu TSUBONE2), Haruka TAKIMOTO ${ }^{1)}$, Daisuke YAMANAKA ${ }^{3)}$, \\ Masayoshi KUWAHARA ${ }^{1)}$ and Koichi ITO ${ }^{3) *}$ \\ 1) Department of Veterinary Pathophysiology and Animal Health, Graduate School of Agricultural and Life Sciences, The University of \\ Tokyo, 1-1-1 Yayoi, Bunkyo-ku, Tokyo 113-8657, Japan \\ 2) Research Center for Food Safety, Graduate School of Agricultural and Life Sciences, The University of Tokyo, 1-1-1 Yayoi, Bunkyo-ku, \\ Tokyo 113-8657, Japan \\ 3) Department of Food and Physiological Models, Graduate School of Agricultural and Life Sciences, The University of Tokyo, 3145 Ago, \\ Kasama, Ibaraki 319-0206, Japan
}

(Received 15 April 2016/Accepted 18 April 2016/Published online in J-STAGE 1 May 2016)

ABSTRACT. Systemic oxidative stress is considered to cause aging. In this study, to estimate the oxidative stress level in senescence-accelerated mouse prone 8 (SAMP8), we evaluated serum reactive oxygen species production and reduction capacity by measurement of Diacron-Reactive Oxygen Metabolites (d-ROM) and Biological Antioxidant Potential (BAP), respectively, with age. SAMP8 showed earlier increase of d-ROM value with age than SAM resistant 1 (SAMR1), the control strain. The BAP level was the highest in adult SAMP8, whereas SAMR1 presented the sustained BAP values between ages. These results indicate that oxidative stress in SAMP8 is higher than SAMR1. Our study is the first detailed report about d-ROM and BAP in SAMP8 and will provide useful fundamental data for future aging studies.

KEY WORDS: aging, BAP, d-ROM, oxidative stress, senescence-accelerated mouse

doi: 10.1292/jvms.16-0204; J. Vet. Med. Sci. 78(8): 1369-1371, 2016

Oxidative stress is generally attributed to imbalance of oxidation-reduction, that is, reactive oxygen species (ROS) production and antioxidant potential, and is associated with a wide variety of diseases, such as arteriosclerosis, diabetes and cancer $[3,10]$. Acceleration of aging is also considered to be caused by systemic oxidative stress $[2,16]$. The senescence-accelerated mouse prone 8 (SAMP8) is one of the strains which shows a short life-span or rapid advancement of senescence, having been used as a representative for aging studies due to exhibiting age-related impairment of learning and memory $[6,11,17]$. Earlier studies have demonstrated that SAMP8 presents elevation of oxidative markers $[1,9$, $12,14,15]$. Recently, new approaches for determination of ROS using an indicator termed Diacron-Reactive Oxygen Metabolites (d-ROM) and reduction capacity using Biological Antioxidant Potential (BAP) measurement at the same time with the same samples have been developed and applied for the veterinary medical science $[4,8,13]$. However, dROM test and BAP test have not been conducted on SAMP8 in detail. Here, we report the d-ROM and BAP values measured quantitatively with age in SAMP8 and evaluated the oxidative stress level in the animal model of aging.

*Correspondence to: Ito, K., Department of Food and Physiological Models, Graduate School of Agricultural and Life Sciences, The University of Tokyo, 3145 Ago, Kasama, Ibaraki 319-0206, Japan. e-mail: akoito@mail.ecc.u-tokyo.ac.jp

(C)2016 The Japanese Society of Veterinary Science

This is an open-access article distributed under the terms of the Creative Commons Attribution Non-Commercial No Derivatives (by-nc-nd) License $<$ http://creativecommons.org/licenses/by-nc-nd/4.0/>.
All animal experiments were performed in accordance with the Institute of Ethical Guidelines under the protocols approved by the Animal Experimental Expert Committee of the University of Tokyo. SAMs were purchased from Japan SLC Inc. (Hamamatsu, Japan). They were housed three to five animals per cage under $12 \mathrm{hr}$ light/dark cycles (lights on at 8:00 am) in a temperature-controlled room $\left(24^{\circ} \mathrm{C}\right)$ and given free access to food and water. We first measured the longevity of SAMs to check if SAMP8 has a short life-span in our experimental environment. We excluded animals which died of obvious infection or injury. As shown in Fig. 1, SAMP8 significantly lived out their natural lives earlier than SAM resistant 1 (SAMR1) which is the control strain of SAMP8. There was no significant difference in the lifespan between male and female (Table 1). These results demonstrate that SAMP8 certainly showed the short lifespan in our experimental environment. Next, we measured the d-ROMs and BAPs from the blood serum of SAMR1 and SAMP8 to check if the oxidative stress increases with age. Male SAMP8 and SAMR1 were used. We collected the whole blood samples from adult (31-37 weeks of age), old (88-94 weeks of age) and aged (115-119 weeks of age) SAMs under deep anesthesia by urethane $(1.0 \mathrm{~g} / \mathrm{kg}$, Sigmaaldrich, St. Louis, MO, U.S.A.). We could not obtain the whole blood samples from 'aged' SAMP8, because of its short life-span. Blood samples were centrifuged at 3,000 rpm for $15 \mathrm{~min}$ at $4{ }^{\circ} \mathrm{C}$ after leaving them undisturbed at room temperature for $1 \mathrm{hr}$. We used the supernatant as serum and measured d-ROM and BAP using a free radical analyzer (FREE carpe diem, WISMERLL, Tokyo, Japan) as described previously [20]. The d-ROM test and BAP test demonstrate 


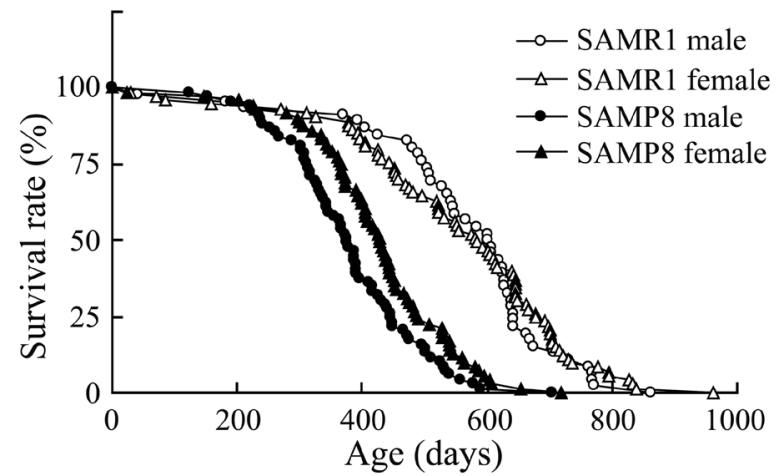

Fig. 1. The longevity of SAMR1 and SAMP8. SAMP8 had a significant short life-span compared with SAMR1. There were no significant differences of longevity between male and female in either SAMR1 or SAMP8.

the hydroperoxide level by detecting absorbance of $\mathrm{N}, \mathrm{N}-$ Diethyl-para-phenylenediamine oxidized by hydroperoxide metabolites, and the antioxidant potential by measuring the reducing capacity of thiocyanate in reducing $\mathrm{Fe}^{3+}$ to $\mathrm{Fe}^{2+}$, respectively $[5,20]$. The d-ROM level of adult SAMP8 was comparable with age-matched SAMR1 (Fig. 2A). However, the d-ROM level of old SAMP8 was significantly higher than not only that of age-matched SAMR1 but also that of adult SAMP8. Furthermore, aged SAMR1 showed higher d-ROM level compared to adult and old SAMR1. To put it simply, the d-ROM level of SAMP8 elevated earlier than SAMR1. SAMP8 presents early attenuation of learning and memory in addition to premature aging [11]. In the previous studies, we have already elucidated that this attenuation of brain function with age is correlated to the hippocampal long-term potentiation (LTP), which is the representative of the synaptic plasticity $[7,18,19]$. The attenuation curve of LTP in SAMP8 shifts earlier than in SAMR1 as with the case of the increase curve of d-ROM which implies hydroperoxide level, indicating that oxidative stress increases with age and it is associated with impairment of LTP and cognitive dysfunction.

The BAP level of adult SAMP8 was the highest among all the groups (Fig. 2B). But, this difference between SAMR1 and SAMP8 disappeared with age due to the decrease of BAP level of SAMP8 and continuous BAP level of SAMR1 for its entire life, indicating that antioxidant capacity decreases with age in SAMP8 but not in SAMR1. Although the values of d-ROM and BAP seem to be inverse relationship in general, that is not necessarily in practice. Our explanation about d-ROM-BAP relationship is as follows; in adult SAMP8, d-ROM level was kept low, because higher BAP level reduced the d-ROM level even if adult SAMP8 had more oxidative stress due to its early senescence. However, as BAP capacity declines in old SAMP8 due to aging, oxidative stress (d-ROM level) in old SAMP8 could not be reduced by BAP, resulting in the higher d-ROM value, whereas old SAMR1 is still in robust health and needs not to increase the BAP level, supported by the fact that d-ROM level is
Table 1. Male and female SAMP8 showed significant short life-spans compared to male and female SAMR1, respectively $(\mathrm{P}<0.01)$. There was no difference in the life-span between sexes. Statistical analysis was carried out using one-way ANOVA followed by Tukey's post hoc test

\begin{tabular}{clcccc}
\hline Strain & Sex & $\mathrm{n}$ & Mean (days) & SD & Median (days) \\
\hline SAMR1 & male & 46 & 567 & 156 & 604 \\
SAMR1 & female & 73 & 556 & 181 & 583 \\
SAMP8 & male & 69 & $381^{* *}$ & 109 & 376 \\
SAMP8 & female & 71 & $426^{* *}$ & 117 & 428 \\
\hline
\end{tabular}

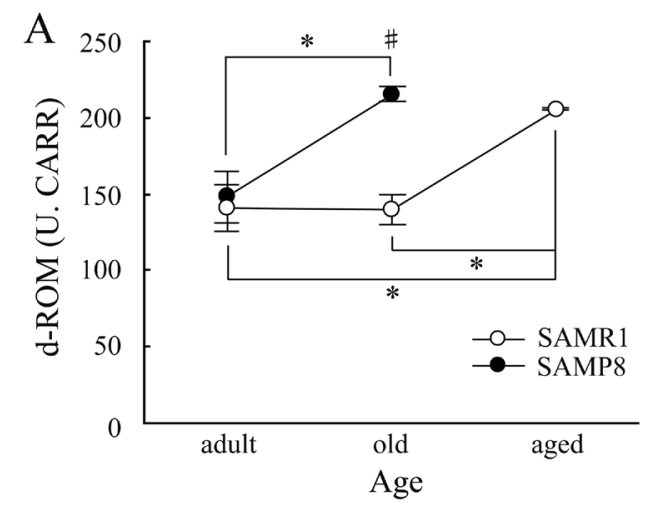

B

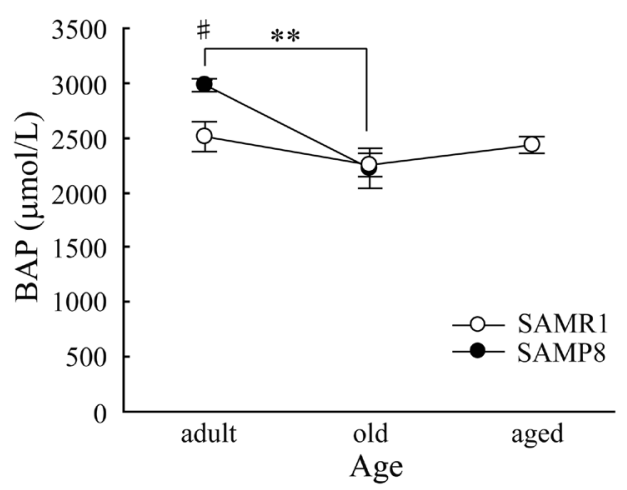

Fig. 2. Age-dependency of d-ROM (A) and BAP (B) levels of SAMR1 and SAMP8. The unit of d-ROM value is U.CARR, an arbitrary unit [20]. Note that no data could be obtained from aged SAMP8 due to its short life-span. All values are represented as mean \pm SE. Statistical analysis was carried out using one-way ANOVA followed by Tukey's post hoc test, and the significant level for the statistical tests was set at 0.01 $(* *)$ or 0.05 (*:among ages, \#:between strains).

comparable with adult SAMR1. Nevertheless, d-ROM level of aged SAMR1 increases due to aging, despite continuous BAP value. This explanation is likely because chronic stress or overwork lowers the BAP level [20], suggesting that BAP level is easily affected by body conditions. As SAMP8 shows early senescence compared to SAMR1, BAP capacity may not function thoroughly, resulting in increase of the dROM value. However, detailed mechanism of d-ROM and BAP relationship should be investigated in the future. 
In summary, we confirmed the longevity of SAMP8 in our experimental condition and measured serum d-ROM and BAP with age to estimate the oxidative stress level in SAMP8. Although the d-ROM level increased with age in both SAMR1 and SAMP8, the increase curve was earlier in SAMP8 than in SAMR1. In contrast, BAP level was the highest in adult SAMP8, whereas SAMR1 presented the sustained BAP values for its entire life. Our study is the first report that reveals age-dependent detailed changes of d-ROM and BAP in SAMP8 and will contribute to future aging studies as useful and fundamental information.

ACKNOWLEDGMENTS. The authors thank Dr. Takashi Matsuwaki in Department of Veterinary Physiology, The University of Tokyo, for helpful advice on this study. There are no conflicts of interest.

\section{REFERENCES}

1. Ali, A. K., Banks, W. A., Kumar, V. B., Shah, G. N., Lynch, J. L., Farr, S. A., Fleegal-DeMotta, M. A. and Morley, J. E. 2009. Nitric oxide activity and isoenzyme expression in the senescenceaccelerated mouse p8 model of Alzheimer's disease: effects of anti-amyloid antibody and antisense treatments. J. Gerontol. A Biol. Sci. Med. Sci. 64: 1025-1030. [Medline] [CrossRef]

2. Conti, V., Izzo, V., Corbi, G., Russomanno, G., Manzo, V., De Lise, F., Di Donato, A. and Filippelli, A. 2016. Antioxidant Supplementation in the Treatment of Aging-Associated Diseases. Front Pharmacol 7: 24. [Medline] [CrossRef]

3. Devarajan, A., Shih, D. and Reddy, S. T. 2014. Inflammation, infection, cancer and all that...the role of paraoxonases. $A d v$. Exp. Med. Biol. 824: 33-41. [Medline] [CrossRef]

4. Fazio, F., Casella, S., Giannetto, C., Caola, G. and Piccione, G. 2009. Serum homocysteine and oxidative stress evaluation during exercise in horse. Pol. J. Vet. Sci. 12: 169-174. [Medline]

5. Fukui, T., Yamauchi, K., Maruyama, M., Yasuda, T., Kohno, M. and Abe, Y. 2011. Significance of measuring oxidative stress in lifestyle-related diseases from the viewpoint of correlation between d-ROMs and BAP in Japanese subjects. Hypertens. Res. 34: 1041-1045. [Medline] [CrossRef]

6. Ito, K. 2013. Frontiers of model animals for neuroscience: two prosperous aging model animals for promoting neuroscience research. Exp. Anim. 62: 275-280. [Medline] [CrossRef]

7. Ito, K., Contractor, A. and Swanson, G. T. 2004. Attenuated plasticity of postsynaptic kainate receptors in hippocampal CA3 pyramidal neurons. J. Neurosci. 24: 6228-6236. [Medline] [CrossRef]

8. Kurokawa, S., Niwano, S., Niwano, H., Ishikawa, S., Kishihara, J., Aoyama, Y., Kosukegawa, T., Masaki, Y. and Izumi, T. 2011. Progression of ventricular remodeling and arrhythmia in the primary hyperoxidative state of glutathione-depleted rats. Circ. J. 75: 1386-1393. [Medline] [CrossRef]

9. Liu, J. and Mori, A. 1993. Age-associated changes in superoxide dismutase activity, thiobarbituric acid reactivity and reduced glutathione level in the brain and liver in senescence accelerated mice (SAM): a comparison with ddY mice. Mech. Ageing Dev. 71: 23-30. [Medline] [CrossRef]

10. Matsuda, M. and Shimomura, I. 2013. Increased oxidative stress in obesity: implications for metabolic syndrome, diabetes, hypertension, dyslipidemia, atherosclerosis, and cancer. Obes. Res. Clin. Pract. 7: e330-e341. [Medline] [CrossRef]

11. Miyamoto, M., Kiyota, Y., Yamazaki, N., Nagaoka, A., Matsuo, T., Nagawa, Y. and Takeda, T. 1986. Age-related changes in learning and memory in the senescence-accelerated mouse (SAM). Physiol. Behav. 38: 399-406. [Medline] [CrossRef]

12. Nomura, Y., Wang, B. X., Qi, S. B., Namba, T. and Kaneko, S. 1989. Biochemical changes related to aging in the senescenceaccelerated mouse. Exp. Gerontol. 24: 49-55. [Medline] [CrossRef]

13. Piccione, G., Giannetto, C., Marafioti, S., Faggio, C., Alberghina, D. and Fazio, F. 2012. Training-induced modifications of circadian rhythmicity of peroxidative parameters in horses. J. Anim. Physiol. Anim. Nutr. (Berl.) 96: 978-984. [Medline] [CrossRef]

14. Sato, E., Kurokawa, T., Oda, N. and Ishibashi, S. 1996. Early appearance of abnormality of microperoxisomal enzymes in the cerebral cortex of senescence-accelerated mouse. Mech. Ageing Dev. 92: 175-184. [Medline] [CrossRef]

15. Sato, E., Oda, N., Ozaki, N., Hashimoto, S., Kurokawa, T. and Ishibashi, S. 1996. Early and transient increase in oxidative stress in the cerebral cortex of senescence-accelerated mouse. Mech. Ageing Dev. 86: 105-114. [Medline] [CrossRef]

16. Sohal, R. S. and Orr, W. C. 1992. Relationship between antioxidants, prooxidants, and the aging process. Ann. N. Y. Acad. Sci. 663: 74-84. [Medline] [CrossRef]

17. Takeda, T., Hosokawa, M. and Higuchi, K. 1991. Senescenceaccelerated mouse (SAM): a novel murine model of accelerated senescence. J. Am. Geriatr. Soc. 39: 911-919. [Medline] [CrossRef]

18. Taniguchi, S., Kuwahara, M. and Ito, K. 2015. Chronic administration of N-acetyl-D-mannosamine improves age-associated impairment of long-term potentiation in the senescence-accelerated mouse. Neurosci. Lett. 598: 41-46. [Medline] [CrossRef]

19. Taniguchi, S., Mizuno, H., Kuwahara, M. and Ito, K. 2015. Early attenuation of long-term potentiation in senescence-accelerated mouse prone 8. Exp. Brain Res. 233: 3145-3152. [Medline] [CrossRef]

20. Tsubone, H., Hanafusa, M., Endo, M., Manabe, N., Hiraga, A., Ohmura, H. and Aida, H. 2013. Effect of Treadmill Exercise and Hydrogen-rich Water Intake on Serum Oxidative and Anti-oxidative Metabolites in Serum of Thoroughbred Horses. J. Equine Sci. 24: 1-8. [Medline] [CrossRef] 